\title{
CYPERUS NIGER Y C. FUSCUS (CYPERACEAE) NUEVAS CITAS PARA CHILE Y APORTES A LA DISTRIBUCIÓN DE CYPERACEAE ALTOANDINAS
}

\author{
Patricio Medina1, Patricio Saldivia² \& Luis Faúndez ${ }^{3,4}$
}

\begin{abstract}
${ }^{1}$ Investigador independiente. Laguna San Rafael 3117, Puerto Varas, Chile; alstroemeds@gmail.com (autor corresponsal). ${ }^{2}$ Department of Botany, University of Otago, Dunedin, New Zealand.

${ }^{3}$ Biota, Gestión y Consultorías Ambientales Ltda. Av. Miguel Claro 1224, Providencia, Santiago, Chile.

${ }^{4}$ Laboratorio de Morfología y Taxonomía Vegetal, Facultad de Ciencias Agronómicas, Universidad de Chile. Av. Santa Rosa 11315, Casilla 1004, La Pintana, Santiago, Chile.
\end{abstract}

\begin{abstract}
Medina, P.; P. Saldivia \& L. Faúndez. 2019. Cyperus niger and C. fuscus (Cyperaceae) new records for Chile and contributions to the distribution of high Andean Cyperaceae. Darwiniana, nueva serie 7(1): 152-166.

The specimens of Cyperaceae present in the main Chilean herbaria (AGUCH, CONC, SGO, and ULS) are scarce and, consequently, information about the chorology of the family is deficient and incomplete at the national level. This work reports two new records of the genus Cyperus for Chile: Cyperus niger, collected in the basin of middle Conay river, in the Atacama Region; and Cyperus fuscus, which is adventitious in America, found near the mouth of the Bío-Bío river, in the Bío-Bío Region. Additionally, new records, localities, and distribution maps that complement the chorology of the following Andean species: Amphiscirpus nevadensis, Eleocharis tucumanensis, Zameioscirpus gaimardioides, and $Z$. muticus in Chile are reported, and the morphology of the latter two discussed in taxonomic terms. Finally, an artificial key to the genera of Cyperaceae in Chile is presented.
\end{abstract}

Keywords. Chile; Cyperus new records; distribution; high Andean Cyperaceae.

Resumen. Medina, P.; P. Saldivia \& L. Faúndez. 2019. Cyperus niger y C. fuscus (Cyperaceae) nuevas citas para Chile y aportes a la distribución de Cyperaceae altoandinas. Darwiniana, nueva serie 7(1): 152-166.

Existe una reducida y poco representativa cantidad de especímenes de herbario de Cyperaceae de Chile en los principales herbarios del país (AGUCH, CONC, SGO y ULS) y, consecuentemente, una carencia de información acerca de la corología de la familia a nivel nacional. En este trabajo se reportan dos nuevas citas del género Cyperus para Chile: Cyperus niger, colectado en la cuenca media del río Conay, Región de Atacama; y Cyperus fuscus, especie adventicia en América, hallada en la ribera norte del río Bío-Bío, cerca de su desembocadura, en la Región de Bío-Bío. Además, se presentan nuevos registros, localidades y mapas de distribución que complementan la corología de las siguientes especies andinas: Amphiscirpus nevadensis, Eleocharis tucumanensis, Zameioscirpus gaimardioides y Z. muticus en Chile; la morfología de las dos especies de Zameioscirpus se discute en términos taxonómicos. Finalmente, se provee una clave artificial para el reconocimiento de los géneros de Cyperaceae distribuidos en Chile.

Palabras clave. Chile; Cyperaceae altoandinas; distribución; nuevos registros de Cyperus.

\section{INTRODUCCIÓN}

La familia Cyperaceae se encuentra representada en Chile por unas 160 especies y unos 10 taxa infraespecíficos (Zuloaga et al., 2008; Rodríguez et al., 2018; Govaerts et al., 2019), distribuidos en 18 géneros presentes en casi todo el territorio nacional (Barros, 1941, 1945; Goetghebeur, 1998; Zuloaga et al., 2008; Rodríguez et al., 2018; Govaerts et al., 2019). 17 géneros (incl. Uncinia Pers. dentro de Carex L., sensu Global Carex Group, 2015) habitan en Chile continental, 
mientras que el género Machaerina Vahl cuenta con una especie endémica de la isla Robinson Crusoe, Archipiélago de Juan Fernández (Strong, 1997; Marticorena et al., 1998a; Bernardello et al., 2006; Moreira-Muñoz, 2012). Por otra parte, el género Cyperus L. se encuentra representado en Chile por 22 especies y 2 variedades (Zuloaga et al., 2008; Rodríguez et al., 2018).

Desde un punto de vista biogeográfico, MoreiraMuñoz (2012) señala que los géneros presentes en Chile forman parte de los siguientes elementos florísticos:

Neotropical: Phylloscirpus C. B. Clarke y Zameioscirpus Dhooge \& Goetghebeur, aunque su distribución se localiza casi completamente a la cordillera de los Andes (Dhooge et al., 2003; Dhooge \& Goetghebeur, 2004a, 2004b; Dhooge, 2005);

Anfitropical: Amphiscirpus Oteng-Yeboah e Isolepis $\mathrm{R}$. Br.;

Pantropical: Carpha Banks \& Sol. ex R. Br., Bulbostylis DC. y Machaerina;

Australasiáticos: Ficinia Schrad. y Oreobolus R. Br.;

Cosmopolita: Bolboschoenus Palla, Carex L. (incl. Uncinia Pers., sensu Global Carex Group, 2015), Cyperus L. (incl. Pycreus P. Beauv.), Eleocharis R. Br., Rhodoscirpus Léveillé-Bourret, Donadío \& J.R.Starr, Rhynchospora Willd., Schoenoplectus (Rchb.) Palla, Schoenus L. y Scirpus L.

La familia ha sido escasamente estudiada en Chile y, consecuentemente, el conocimiento de su distribución en el territorio resulta fragmentado e incompleto (Marticorena, 1990; Marticorena et al., 1998b; Squeo et al., 2001, 2008; Dhooge, 2005; Zuloaga et al. 2008; García, 2013; Gatica-Castro et al., 2015; Rodríguez et al., 2018; Govaerts et al., 2019).

El objetivo principal de esta nota es reportar el hallazgo de Cyperus niger y C. fuscus como nuevos registros para Chile, y actualizar el rango de distribución dentro del país de cuatro Cyperaceae altoandinas. Se incrementa la cantidad de ejemplares en los herbarios AGUCH, EIF, CONC y SGO, se presentan mapas de su distribución y, de manera adicional, se proporciona una clave taxonómica artificial para el reconocimiento de los géneros de Cyperaceae presentes en Chile.

\section{MATERIALES Y MÉTODOS}

Cyperus niger fue registrado durante una exploración botánica en marzo de 2011, en la cuenca media del río Conay, comuna de Alto del Carmen, provincia de Huasco, Región de Atacama. Cyperus fuscus fue recolectado en marzo de 2016, tras recorrer la ribera norte del río Bío-Bío, en el sector próximo a su desembocadura en el Océano Pacífico, comuna de Hualpén, provincia de Concepción, Región del Bío-Bío.

Durante diversas exploraciones botánicas dirigidas a vegas y humedales de altura, entre los años 1990 y 2017, se recolectó material de Cyperaceae altoandinas de los géneros Amphiscirpus, Zameioscirpus y Eleocharis; estas se extendieron desde el límite norte de Chile, en la Región de Arica y Parinacota, hasta la Región del Maule en el centro-sur. El material recolectado se depositó en los herbarios AGUCH y CONC.

La determinación taxonómica de las especies, se basó en la literatura acorde a cada taxón: Amphiscirpus nevadensis (Barros, 1969; Smith, 2002; Dhooge, 2005; Guaglianone et al., 2009), Zameioscirpus (Dhooge et al., 2003, 2004; Dhooge, 2005; Guaglianone et al., 2009), Eleocharis tucumanensis (Guaglianone et al., 1998; Guaglianone \& González-Elizondo, 2009). Cyperus niger (Barros, 1938; Corcoran, 1941; Tucker et al., 2002; Guaglianone et al., 2009). Cyperus fuscus (Kükenthal, 1935-1936; Costé, 1937; McGivney 1938; Tucker et al. 2002; Castroviejo, 2008; Dai et al., 2010). Se revisaron los protólogos y especímenes tipo digitalizados de GENT, K, MA, SGO, SI y UPS, a través de la plataforma “Global Plant Initiative, GPP” (http://plants.jstor. org) y fotografías de especímenes digitalizados disponibles en catálogos en línea de los herbarios E, GH, K, US y WU. También, se consultaron especímenes depositados en AGUCH, CONC, EIF, SGO y ULS, junto con información de especímenes señalados en la literatura taxonómica (e.g. Dhooge et al., 2003, 2004; Dhooge, 2005). El ordenamiento taxonómico sigue principalmente al del Catálogo de la flora vascular del Cono Sur (Zuloaga et al., 2008, constantemente actualizado), Catálogo de la flora de Chile (Rodríguez et al., 2018) y "World Checklist of Selected Plant Families" (Govaerts et al., 2019). La nomenclatura de los acrónimos de 
los herbarios consultados sigue a Thiers (2019). La elaboración de la clave de géneros se basó en Barros (1945, 1969), Adams (1994), Goetghebeur \& Simpson (1991), Goetghebeur (1998), Ball et al. (2002), Luceño et al. (2008); Tucker et al. (2002) y Guaglianone et al. (2009), junto con observaciones macro morfológicas realizadas bajo microscopio estereoscópico marca Ivens, modelo XTL-3 con aumento tipo zoom regulable de 4,5x y aumento ocular de 10x. Los mapas de distribución se elaboraron sobre la base de material con referencia espacial disponible, mediante software SIG de libre acceso QGIS versión 2.18 (QGIS, 2019).

\section{RESULTADOS}

\section{Nuevas citas del género Cyperus L. para Chile}

Cyperus fuscus L. Sp. pl. 1:46. 1753. TIPO: Francia, Monspelii. Lipsiae "item in ipsa scaturigine thermarum Vallesianarum", 16001625, J. Burser s.n. (lectotipo UPS-HERB. BURSER 1:82!, designado por Kukkonen, Taxon 53: 179. 2004). Fig.1a-c.

Nombres vernáculos. No se registran en Chile; en Estados Unidos y Canadá se conoce como "brown galingale" (Tucker et al., 2002; Lansdown, 2013); Europa (España y Portugal) como "juncia", "serrana", "serrana fosca" (Castroviejo, 2008).

Referencias. Boeckeler, (1867: 586), Kükenthal (1935-1936: 235), Costé (1937: 461), McGivney (1938: 42), Tucker et al. (2002: 157), Castroviejo (2008: 19), Dai et al. (2010: 225).

Iconografía. Costé (1937: 461), McGivney (1938: 66, plate VIII), Tucker et al. (2002), Dai et al. (2010: 301, fig. 8-11).

Distribución geográfica y hábitat. Distribución natural en Europa centro-mediterránea, suroeste de Asia (China, India), Macaronesia, norte de África; adventicia en Norteamérica (Costé, 1937; Tucker et al., 2002; Castroviejo, 2008; Dai et al., 2010; Larridon et al., 2011; Lansdown, 2013; Govaerts et al., 2019). Maleza asociada a cultivos de arroz, sitios pantanosos, riberas, desembocaduras, zonas encharcadas y canales (McKenzie et al., 1998; Tucker et al., 2002; Castroviejo, 2008; Dai et al., 2010), desde el nivel del mar y hasta los $2000 \mathrm{~m} \mathrm{~s}$. $\mathrm{m}$. (Castroviejo, 2008, Dai et al., 2010). La ocurrencia en Chile fue registrada para la ribera norte del río Bío-Bío, cerca de su desembocadura (Fig. 2). El hábitat se caracteriza por presentar una fisiografía plana, elevación media de $6 \mathrm{~m} \mathrm{s.} \mathrm{m}$. y micro relieve irregular generado por crecidas frecuentes del río. El sustrato posee textura arenosa, es húmedo y está sujeto a procesos de arrastre-depósito de sedimentos generado por el régimen fluvial exorreico del río.

La comunidad de la que forma parte, está dominada por especies herbáceas de origen alóctono características de ambientes modificados. Las especies dominantes corresponden a Cyperus squarrosus L., Eragrostis mexicana subsp. virescens (J. Presl) S.D. Koch \& Sanchéz Veg, Melilotus albus Desr. y Salix viminalis L.; como especies asociadas están: Alisma plantago-aquatica L., Cynodon dactylon (L.) Pers., Dysphania multifida L., Lotus pedunculatus Cav., Polygonum persicaria L., Xanthium strumarium L., junto con escasas especies nativas como el arbusto Baccharis salicifolia (Ruiz \& Pav.) Pers., las hierbas perennes Eleocharis pachycarpa E. Desv., Ludwigia peploides (Kunth) P.H. Raven y las hierbas anuales Pseudognaphalium aldunateoides (J. Rémy) C. Monti, Bayón \& S.E. Freire y P. cymatoides (Kunze ex DC.) Anderb.

Categoría de conservación. "Least Concern" (LC), dentro de su área de distribución natural (Lansdown. 2013).

Observaciones. Cyperus fuscus, se diferencia de C. squarrosus, especie anual con mayor afinidad morfológica en Chile, porque en la primera el ápice de la gluma es brevemente apiculado, la carena verde, trinervada, bien marcada y de color purpúreo a pardo oscuro, mientras que, en la segunda, la gluma es largamente apiculada, recurvada, de color rojizo y con numerosos (más de 3 ) nervios notorios.

\section{Material representativo examinado}

CHILE. Región de Bío-Bío. Concepción, ribera del río Bío-Bío cerca de desembocadura al mar. $36^{\circ} 48^{\prime} 44,01^{\prime \prime} \mathrm{S} 73^{\circ} 6^{\prime} 37,58^{\prime \prime} \mathrm{W}, 6$ m s. m., 14-III-2016, Medina 3407 (AGUCH!, CONC!). ESTADOS UNIDOS DE AMERICA. Cooper Co., 

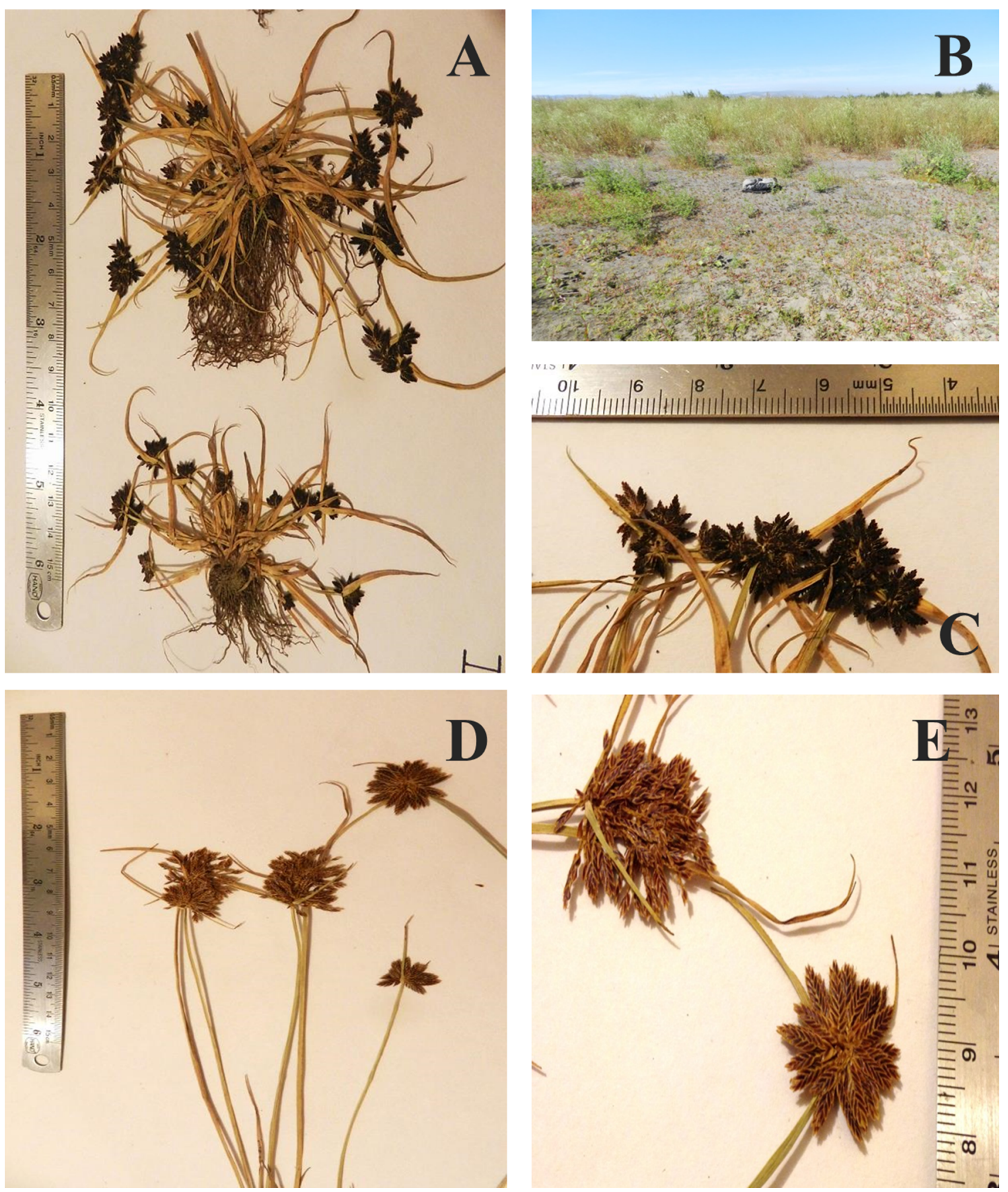

Fig. 1. Cyperus fuscus. A, aspecto general. B, hábitat donde fue registrada la especie. C, detalle inflorescencia. Cyperus niger. D, aspecto general. E, detalle de inflorescencia. A-C de Medina 3407 (CONC); D-E de Medina 3347 (CONC). Fotografías de P. Medina. Figura en color en la versión en línea http://www.ojs.darwin.edu.ar/index.php/darwiniana/article/view/800/1158

Overton Bottoms-big muddy national fish and wildlife refuge, ca. 1.2 miles NNE of Overton, 06-
X-2001, McKenzie 1976 (MO 5318907!, imagen); Boone Co., Mc Baine, West of Columbia waste- 
water treatment lagoon \#3, 13-X-1997, Jacobs 97-37 (MO 5551569!, imagen). TURQUÍA. Sapancagolu Lake, 3km W. of Saracoglu Motel, 40 m s. m., 01-IX-1972, Uotila 20182 (E cb 352637!). $500 \mathrm{~m}$ ostlich der Euphratbrucke zwischen Elazig und Pertek, Sudufer Sandbank am Flubufer, $900 \mathrm{~m}$ s. m., 21-VII-1973, Holtz et al. 87 (E cb 352611!). YEMEN. Wadi at Ad Dabab, c.10 km SW of Taiz, 11-III-1984, Miller \& King 5057 (E cb 352846!).

Cyperus niger Ruiz \& Pav. Fl. Peruv. 1: 47. 1798. TIPO: Perú, Chancay prope Huanam, 12/62, Ruiz \& Pavón s.n. (holotipo MA cb 810290!). Fig. 1d-e.

Referencias. Boeckeler (1867: 454, 456), Kükenthal (1935-1936: 343), MacBride (1936: 274), Barros (1938: 269-270), Corcoran (1941: 5), Adams (1994), Tucker (1994: 76), Tucker et al. (2002: 161), Guaglianone et al. (2009: 298).

Iconografía. Barros (1938: 271, fig. 7), Tucker (1994: 67, fig. 23f), Tucker et al. (2002), Guaglianone et al. (2009: 298, fig. 262).

Distribución geográfica y hábitat. Posee una amplia distribución en América tropical y subtropical, que comprende desde el sur de América del Norte hasta la Argentina (Corcoran, 1941; Tucker, 1983, 1994; Guaglianone, 1996; Tucker et al., 2002; Zuloaga et al., 2008; Guaglianone et al., 2009). Habita en zonas montañosas (Barros, 1938; Guaglianone et al. 2009), pastizales montanos y sub-montanos, en pantanos y en otras áreas abiertas, muy húmedas (Adams, 1994; Tucker et al., 2002), entre 200 - 3200 m s. m. (Guaglianone et al., 2009). Los puntos de recolección más cercanos al sitio de registro en Chile corresponden a los especímenes argentinos L. Goldsack s.n. (SGO 46222) y R. A. Philippi s.n. (SGO-37737), ambos provenientes de la provincia de Mendoza, $R$. Kiesling \& Meglioli 6664 (SI), R. Guaglianone 1616, 1627 (SI), de la provincia de San Juan (Guaglianone et al., 2009). En Chile, la especie fue registrada en la ribera del río Chollay (1400 m s. m.), afluente del río Conay y en los alrededores de los ríos Del Carmen (aguas arriba del río Huasco, $790 \mathrm{~m} \mathrm{~s}$. m.) y río del Chañarcillo (2053 m s. m.); todos los sitios están ubicados en la comuna de Alto del Carmen, provincia de Huasco, Región de Atacama (Fig. 2). Cyperus niger integra una comunidad riparia formada por elementos nativos asociados a cursos de agua, cuyas especies dominantes son: Baccharis salicifolia (Ruiz \& Pav.) Pers., Cortaderia speciosa (Nees) Stapf, Escallonia angustifolia C. Presl. y Tessaria absinthioides (Hook. \& Arn.) DC., las que comparten el espacio con hierbas palustres como Eleocharis cfr. bonariensis Nees, E. dombeyana Kunth, E. pseudoalbibracteata S. González \& Guagl., Polypogon australis Brongn., Triglochin concinna Burtt Davy y plantas acuáticas como Myriophyllum quitense Kunth, varias algas Chlorophyceae y del género Chara L. Asociadas a esta comunidad, además, se encuentran especies alóctonas asilvestradas, características de ambientes alterados, tales como Agalinis linarioides (Cham. \& Schltdl.) D'Arcy, Melilotus albus Desr., Polygonum persicaria L., Polypogon monspeliensis (L.) Desf., Veronica Anagallis-aquatica L. y Salix babylonica L.

Categoría de conservación. Se dispone de escasos registros de la especie en Chile y no existe información consistente acerca de potenciales amenazas. Se sugiere la categoría Datos Insuficientes (DD).

Observaciones. Cyperus niger se diferencia de otros Cyperus presentes en Chile por sus espiguillas de color pardo oscuro y por sus aquenios lateralmente comprimido-lenticulares, dispuestos de manera lateral enfrentando la raquilla y la bráctea (gluma), debido a esta característica ha sido ubicado en el género Pycreus (Corcoran, 1941; Koyama, 1974; Guaglianone et al., 2009).

\section{Material representativo examinado}

ARGENTINA. Córdoba. Sierra chica de Córdoba, Colachanga, II-1882, Hieronymus s. $n$. (K cb 632078!); IV-1871, Lorentz 31 (CORD); II1872, Lorentz 36 (CORD). Mendoza. Mendoza, I/II-1885, Goldsack s. n. (SGO 46222!); ídem, 1868/1869, Philippi s. n. (SGO 37737!); ídem, 1868/1869, Philippi s. n. (SGO 46255!, 37741!). BOLIVIA. La Paz. Manco Kapac, Isla del Sol La Paz, 3820 m s. m., 24-I-1986, Liberman 1210 (LPB, NY). CHILE. Región de Atacama. Huasco, ribera de río Chollay aguas arriba confluencia río Conay, 2858'23,04'S 70 9'25,53”W, $1417 \mathrm{~m} \mathrm{~s}$. m., III-2011, Medina 3347 (AGUCH!, CONC!). 



Fig. 2. Mapa de distribución en Chile de Cyperus niger (triángulo), C. fuscus (círculo), Amphiscirpus nevadensis (estrella) y Eleocharis tucumanensis (rombos). Figura en color en la versión en línea http://www.ojs.darwin.edu.ar/ index.php/darwiniana/article/view/800/1158

\section{Aportes a la distribución de Cyperaceae altoandinas en Chile}

Amphiscirpus nevadensis (S.Watson) OtengYeb., Notes Roy. Bot. Gard. Edinurgh 33 (2): 308. 1974. Scirpus nevadensis S.Watson, Botany [Fortieth Parallel] 360. 1871. TIPO: Estados Unidos de América, Nevada, Soda Lake, Carson Desert, S. Watson 1213 (holotipo US; isotipos GH cb 28028!, NY cb 00051647!, YU cb 001025!).

Nombres vernáculos. En Chile y Argentina se la conoce como "unquillo" (Villagrán \& Castro, 2004; Guaglianone et al., 2009).

Referencias. Barros (1969), Oteng-Yeboah (1974), Goethgebeur \& Simpson (1991), Goetghebeur (1998), Smith (2002), Dhooge (2005), Guaglianone et al. (2009).
Distribución geográfica y hábitat. Se distribuye en ambos hemisferios del continente americano, desde Canadá hasta la Patagonia de Argentina y Chile, principalmente en ambientes de montaña, hasta los 4000 m s.m. (Barros, 1969; Oteng-Yeboah, 1974; Goetghebeur \& Simpson, 1991; Guaglianone, 1996; Goetghebeur, 1998; Smith, 2002; Dhooge, 2005; Zuloaga et al., 2008, Guaglianone et al., 2009; Rodríguez et al., 2018; Govaerts et al., 2019). Para Sudamérica ha sido citada para Argentina, Bolivia y Chile (Goetghebeur, 1998; Dhooge, 2005; Zuloaga et al., 2008; Guaglianone et al., 2009; Jørgensen et al. 2014; Govaerts et al., 2019). Pese a su amplia distribución general, esta especie ha sido escasamente registrada y citada para Chile, con reducidos ejemplares de herbario y a su vez localidades de recolecta (Dhooge, 2005). Gatica-Castro et al. (2015) señalan que la especie 
se distribuye en Chile en los extremos norte (Regiones de Arica y Parinacota y Tarapacá) y sur (Región de Magallanes), aunque no se detallan especímenes de herbario ni localidades. Alfaro y Faúndez (2010) mencionan la presencia de la especie en una vega salina en el salar de Aguas Calientes (Región de Antofagasta), registro que es confirmado en este trabajo (Faúndez y Alfaro s.n. AGUCH). De manera adicional, existe un material de R. A. Philippi proveniente de Atacama (K cb 881756 !), aunque sin mayores detalles de localidad. El límite sur de distribución en Chile alcanza la Región de Magallanes, en el Parque Nacional Pali Aike (Domínguez 157, CONC 159081). Fig. 2.

Categoría de conservación. Sin protección en la normativa de Chile. No ha sido sometida al proceso de evaluación definido por el Reglamento de Clasificación de Especies del Ministerio de Medio Ambiente de Chile. Clasificada en la propuesta de estados de conservación de la flora de las regiones de Arica y Parinacota y de Tarapacá bajo la categoría "Datos Insuficientes" (GaticaCastro et al., 2015).

\section{Material representativo examinado}

CHILE. Región de Antofagasta. El Loa, laguna Tujajtu sector Aguas Calientes, 235'28'S 67³9’44,8”W, 3946 m s. m., 18-XII-2013, Saldivia 2185 (AGUCH!); El Loa, Salar aguas calientes, 235'28,91'S 67³9'44,36”'W, 3945 m s. m., 03V-2010, Faúndez y Alfaro s. n. (AGUCH!). Región de Atacama. Atacama, II-1888, R. A. Philippi s.n. (K cb 00881756!). Región de Magallanes. Magallanes, Parque Nacional Pali Aike sector laguna Ana, 52 ${ }^{\circ}$ 'S 694' W, 90 m s. m., I-2004, Domínguez 157 (CONC 159081!).

Eleocharis tucumanensis Barros, Lilloa 12: 7. 1946. TIPO: Argentina, Tucumán, Tafí, río de La Puerta, 4000 m s.m., XII-1931, R. Schreiter 7071 (holotipo LIL cb 10323!, isotipo SI cb 61001 !).

Nombres vernáculos. No conocido en Chile; este tipo de hierbas propias de humedales altoandinos se las denomina colectiva o genéricamente "grama", vegas o "t'champa" (Villagrán \& Castro, 2004).
Referencias. Guaglianone et al. (1998), Guaglianone \& González-Elizondo (2009) y Medina (2015).

Distribución geográfica y hábitat. Habita en márgenes de vegas en forma de "cojín” y humedales altoandinos hasta los $4900 \mathrm{~m} \mathrm{~s}$. m., propios de la puna seca de Argentina, Bolivia y Chile (Guaglianone et al., 1998; Guaglianone et al., 2009; Jørgensen et al. 2014; Rodríguez et al., 2018), aunque para Chile es una especie escasamente estudiada y gran parte de la información y material colectado proviene de Argentina (Barros, 1946; Guaglianone, 1996; Guaglianone et al., 1998; Guaglianone y González-Elizondo, 2009). En Chile se distribuye desde la Región de Arica y Parinacota, en el extremo norte (Guaglianone, 1996; Guaglianone et al., 1998; Marticorena et al., 1998b; Guaglianone \& González-Elizondo, 2009; García, 2013; Rodríguez et al., 2018). Recientemente se amplió su distribución a comunidades azonales de alta montaña en la Región Metropolitana de Santiago, Chile Central (Medina, 2015). Fig. 2.

Categoría de conservación. Sin protección en la normativa de Chile. No ha sido sometida al proceso de evaluación definido por el Reglamento de Clasificación de Especies del Ministerio de Medio Ambiente de Chile. Para la Región de Antofagasta fue clasificada bajo el estatus "Datos Insuficientes" (García, 2013). Las principales amenazas para las comunidades donde habita consisten en la extracción de agua para minería a gran escala, que destruye y deseca las comunidades azonales altoandinas y, por consecuencia, produce fragmentación y destrucción de su hábitat. La práctica tradicional de ganadería extensiva podría generar daños en la vegetación por sobrepastoreo y la incorporación de especies ruderales.

\section{Material representativo examinado}

CHILE. Región Metropolitana de Santiago. Prov. Cordillera, vega acojinada alto Andina en estero Los Nacimientos, 3309'55'S 7009'57'W, 3812 m s. m., 09-III-2015, Medina 2900 (SGO!, CONC!); Ídem, 13-IV-2015, Medina 3030 (SGO!, CONC!). Región de Arica y Parinacota. Prov. Parinacota, lago Chungará orilla de laguna, $18^{\circ} 18^{\prime} \mathrm{S}$ $69^{\circ} 08^{\prime} \mathrm{W}, 4600$ m s. m., 1-XI-1991, Ruthsatz 7989 (SI!); Caquena, $18,0660^{\circ} \mathrm{S} 69,1923^{\circ} \mathrm{W}$, 
4442 m s. m., Saldivia 423 (AGUCH!); Itiza, bofedal, $18^{\circ} 43^{\prime} 55^{\prime}$ 'S 69²0'05'W, $3972 \mathrm{~m} \mathrm{s.} \mathrm{m.,}$ 25-IX-2015, Gamboa et al. 2 (SGO!). Salar de Surire, bofedal colgante cerca de Garita CONAF, 18 51'16”'S 6908'34”'W, 4290 m s. m., 24IX-2015, Gamboa et al. 1 (SGO!). Región de Tarapacá. Prov. Del Tamarugal, Collaguasii Ujima en vega, 2059'S, 68³9'W, 4450 m s. m., 24-III1992, Ruthsatz 8423 (SI!); Lirima, $19,8306^{\circ} \mathrm{S}$ 68,8418 W, 4143 m s. m., 14-X-2009, Faúndez et al. s. n. (AGUCH!), $19,8558^{\circ} \mathrm{S} 68,9172^{\circ} \mathrm{W}$, 3992 m s. m., 13-X-2009, Faúndez et al. s. $n$. (AGUCH!). Región de Antofagasta. Prov. El Loa, vega en quebrada Linzor, $22^{\circ} 13^{\prime} \mathrm{S}, 68^{\circ} 01^{\prime} \mathrm{W}$, 4000 m s. m., 21-III-1992, Ruthsatz 8374 (SI!). Región de Atacama. Río Astaburoaga, $27,5908^{\circ} \mathrm{S}$ $69,0775^{\circ} \mathrm{W}, 4496$ m s. m., 25-II-2010, Saldivia 565, $565 b$ (AGUCH!). Región de Coquimbo. Prov. Choapa, quebrada Hualtatas, $31,7111^{\circ} \mathrm{S}$ 70,5190 W, 3560 m s. m., 15-III-2005, Faúndez et al. s. $n$. (AGUCH!). Prov. Limarí, cuenca quebrada larga río Mostazal, $30,8447^{\circ} \mathrm{S} 70,3139^{\circ} \mathrm{W}, 3433 \mathrm{~m}$ s. m., Sánchez et al. s. n. (AGUCH!).

\section{Zameioscirpus gaimardioides (E. Desv.)} Dhooge \& Goetgh., Pl. Syst. Evol. 243: 82. 2003. Carex gaimardioides E. Desv., Fl. Chil. 6: 202. 1853. TIPO: Chile, Coquimbo, C. Gay s.n. (holotipo $\mathrm{P}$ !; isotipos $\mathrm{K}$ cb 000881759 !, $\mathrm{P}$ cb 881759!).

Nombres vernáculos. No conocido en Chile; a este tipo de hierbas propias de humedales altoandinos se les denomina colectiva o genéricamente " $t$ 'champa, t'champita” o vega (Villagrán \& Castro, 2004).

Referencias. Dhooge (2005), Guaglianone et al. (2009).

Distribución geográfica y hábitat. Se encuentra en las regiones andinas de Perú, Argentina y Chile, en elevaciones que fluctúan entre 3000 4800 m s. m. (Guaglianone, 1996; Dhooge, 2005; Zuloaga et al., 2008). En la Argentina ha sido descrita en vegas andinas entre las provincias de Jujuy hasta San Juan (Guaglianone, 1996; Dhooge et al., 2003; Dhooge, 2005; Zuloaga et al., 2008; Guaglianone et al., 2009). Govaerts et al. (2019) señalan la ocurrencia de la especie en la provincia de Neuquén. En Chile se la ha citado por Dhooge et al. (2003) y Dhooge (2005) para humedales altoandinos de la Región de Coquimbo (C. Gay s.n. holotipo K cb 00881758! y Ruthsatz 8930 GENT). De manera adicional, Rodríguez et al. (2018), indican su presencia en la Región de Atacama y La Región del Libertador Bernardo O'Higgins. No obstante, el presente trabajo cita material de herbario referenciado y amplía de manera considerable el rango de distribución en Chile desde la Región de Atacama hasta la Región del Maule (Fig. 3), donde $Z$. gaimardioides forma parte de la vegetación azonal característica de humedales de andinos y en específico vegas acojinadas (Luebert \& Gajardo, 2004; Luebert \& Pliscoff, 2006). Los nuevos registros adicionados concuerdan con el rango de presencia latitudinal que posee la especie en Argentina a lo largo de la cordillera de Los Andes (Guaglianone, 1996; Zuloaga et al., 2008; Guaglianone et al., 2009; Govaerts et al., 2019).

Categoría de conservación. Sin protección en la normativa de Chile. No ha sido sometida al proceso de evaluación definido por el Reglamento de Clasificación de Especies del Ministerio de Medio Ambiente de Chile. No ha sido incorporada en los libros rojos de las regiones de Atacama (Squeo et al., 2008), Coquimbo (Squeo et al., 2001) y de O'Higgins (Serey et al., 2007).

\section{Material representativo examinado}

CHILE. Región de Atacama. Prov. Copiapó, cuenca superior quebrada Caspiche, $27,7436^{\circ} \mathrm{S}$ 69,3568 W, 4266 m s. m., XII-2009, Faúndez s. $n$. (AGUCH!); río Valle Ancho, ante junta, $27,2627^{\circ} \mathrm{S}$ 69,0027 W, 4137 m s. m., XII-2009, Faúndez et al. s. n. (AGUCH!); lagunilla proyecto Caspichal, $27,6905^{\circ} \mathrm{S} 69,2863^{\circ} \mathrm{W}, 4451 \mathrm{~m}$ s. m., $25-\mathrm{X}-2009$, Faúndez s. n. (AGUCH!); estero Barros Negros cercano a estación DGA, $27,2677^{\circ} \mathrm{S} 69,0012^{\circ} \mathrm{W}$, 4096 m s. m., XI-2010, Medina 3324, 3325 (AGUCH 71237!, CONC!); ídem, 27,2677 $\mathrm{S}$ 69,0012 ${ }^{\circ} \mathrm{W}, 4096 \mathrm{~m}$ s. m., II-2011, Medina 3326, 3327 (AGUCH 71236!, CONC!); ídem, 27,2677 $\mathrm{S}$ 69,0012 ${ }^{\circ} \mathrm{W}, 4096 \mathrm{~m} \mathrm{~s} . \mathrm{m} .$, IV-2011, Medina 3328, 3329 (AGUCH 71235!, CONC!); paso Caballo Muerto $26,8221^{\circ} \mathrm{S} 69,1783^{\circ} \mathrm{W}, 4080 \mathrm{~m}$ s. m., IV-2011, Medina 3330, 3331 (AGUCH 71234!, CONC!); ídem, $26,8291^{\circ} \mathrm{S} 69,1686^{\circ} \mathrm{W}, 4006$ m s. m., 

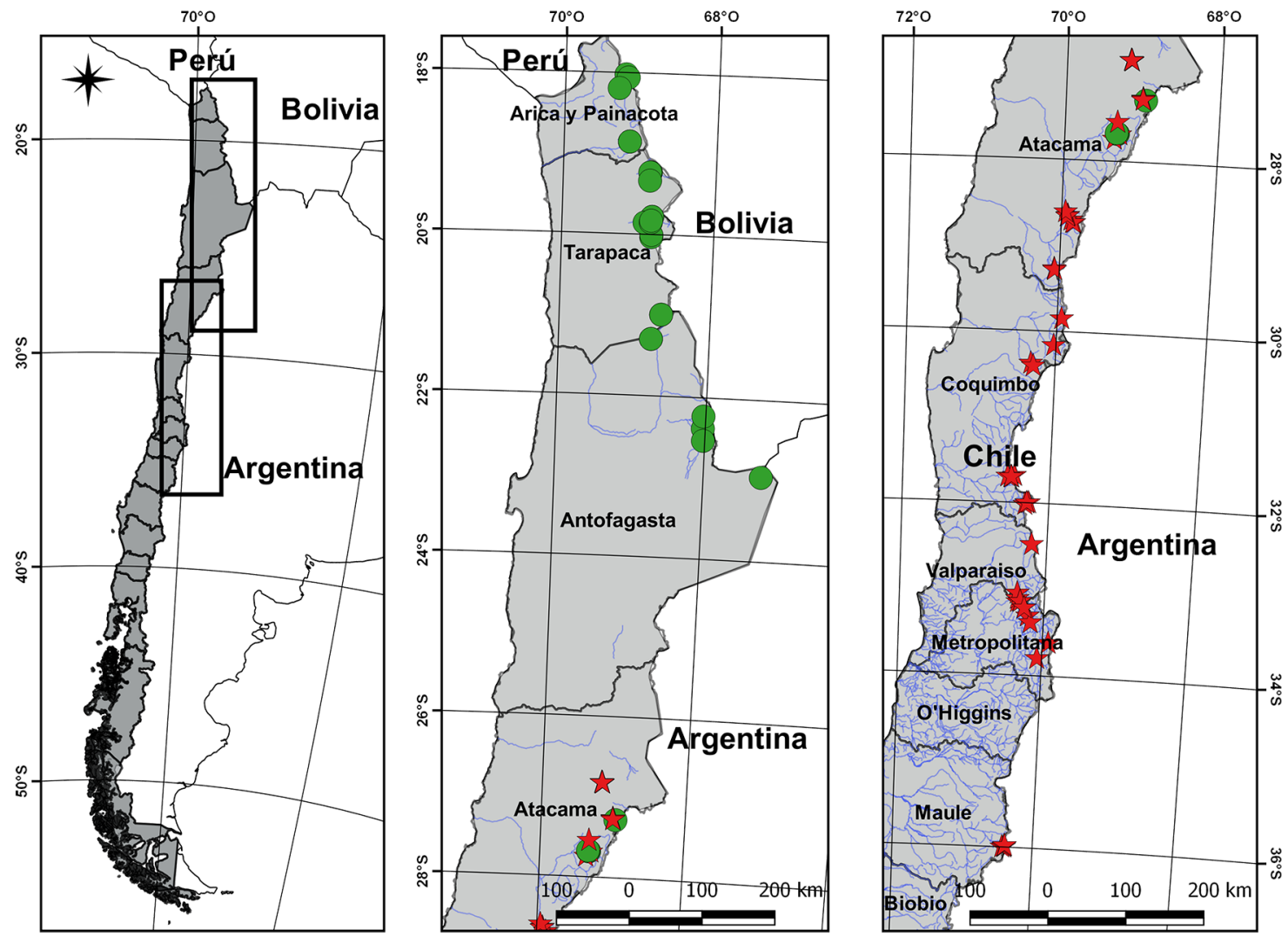

Fig. 3. Mapa de distribución en Chile de Zameioscirpus gaimardioides (estrella) y Z. muticus (círculo). Figura en color en la versión en línea http://www.ojs.darwin.edu.ar/index.php/darwiniana/article/view/800/1158

12-V-2016, Medina 3336, 3337 (AGUCH 71231!, CONC!); ídem, $26,8253^{\circ} \mathrm{S} 69,1738^{\circ} \mathrm{W}, 4000 \mathrm{~m}$ s. m., 22-V-2016, Medina 3338, 3339 (AGUCH 71230 !, CONC!); estero La Laguna (Mina Refugio), 27,5466 ${ }^{\circ} \mathrm{S} 69,3206^{\circ} \mathrm{W}, 4071 \mathrm{~m}$ s. m., 27-XII-2006, Faúndez s. n. (AGUCH!). Prov. Huasco, río Del Estrecho aguas arriba confluencia río Los Barriales, $29,2743^{\circ} \mathrm{S} 70,0676^{\circ} \mathrm{W}, 3744 \mathrm{~m}$ s. m., IX-2010, Medina 3309 (AGUCH 71244!); río Del Estrecho sector nacimiento, $29,2857^{\circ} \mathrm{S} 70,0622^{\circ} \mathrm{W}, 3854$ m s. m., IX-2010, Medina 3341 (CONC!); Alto Huasco quebrada Cantarito (afluente de Laguna grande), $28,7021^{\circ} \mathrm{S} 69,8719^{\circ} \mathrm{W}, 3500 \mathrm{~m}$ s. m., I-1983, Marticorena et al. 83479 (CONC 55179!); ídem, $28,7021^{\circ} \mathrm{S} 69,8719^{\circ} \mathrm{W}, 3500 \mathrm{~m} \mathrm{~s} . \mathrm{m} ., \mathrm{I}-1983$, Marticorena et al. 83508 (CONC 55205!); ídem, $28,7317^{\circ} \mathrm{S} 69,8347^{\circ} \mathrm{W}, 3400 \mathrm{~m}$ s. m., I-1983, Marticorena et al. 83531 CONC 55225!); ídem, $28,7317^{\circ} \mathrm{S} 69,8510^{\circ} \mathrm{W}, 3100 \mathrm{~m}$ s. m., I-1983, Marticorena et al. 83437 (CONC 55139!); cuenca del Tránsito quebrada Larga, $28,6500^{\circ} \mathrm{S} 69,9167^{\circ} \mathrm{W}$, 3950 m s. m., II-2002, Teillier 5025 (GENT); quebrada Larga cerca mina La Fortuna, $28,6587^{\circ} \mathrm{S}$ $69,9243^{\circ} \mathrm{W}, 3754 \mathrm{~m}$ s. m., 05-X-2017, Medina 3600 (CONC!); quebrada Los Piuquenes, $28,6097^{\circ} \mathrm{S}$ $69,9491^{\circ} \mathrm{W}, 3829 \mathrm{~m} \mathrm{s.} \mathrm{m.,} \mathrm{05-X-2017,} \mathrm{Medina} 3601$ (CONC!). Región de Coquimbo. Prov. Choapa, quebrada Hualtatas, $31,7111^{\circ} \mathrm{S} 70,5190^{\circ} \mathrm{W}, 3560 \mathrm{~m}$ s. m., 19-III-2005, Faúndez et al. s. n. (AGUCH!); ídem, $31,7059^{\circ} \mathrm{S} 70,5309^{\circ} \mathrm{W}, 3665 \mathrm{~m} \mathrm{~s} . \mathrm{m} ., 15-$ III-2005, Faúndez et al. s. n. (AGUCH!); ídem, $31,7067^{\circ} \mathrm{S} 70,5254^{\circ} \mathrm{W}, 3623 \mathrm{~m}$ s. m., III-2010, Medina 3320 (AGUCH 71239!); vega el Tizón Cordillera Cuncumén, $32,0203^{\circ} \mathrm{S} 70,3267^{\circ} \mathrm{W}, 3344$ m s. m., 5-IV-2009, Saldivia PS-3 (AGUCH!); cerro Amarillo vega acojinada, $31,6892^{\circ} \mathrm{S} 70,4830^{\circ} \mathrm{W}$, 
3588 m s. m., III-2010, Medina 3312 (AGUCH 71243 !); ídem, $31,6893^{\circ} \mathrm{S} 70,4838^{\circ} \mathrm{W}, 3598 \mathrm{~m} \mathrm{~s}$. m., III-2010, Medina 3342 (CONC!); Vega el Circo, $32,0026^{\circ} \mathrm{S} 70,3000^{\circ} \mathrm{W}, 3433 \mathrm{~m}$ s. m., III-2010, Medina 3314, 3315 (AGUCH 71242!, CONC!); alto Piuquenes, $31,7004^{\circ} \mathrm{S} 70,5746^{\circ} \mathrm{W}, 3283 \mathrm{~m} \mathrm{~s}$. m., III-2010, Medina 3343 (CONC!); laguna el Pelao pequeña vega en su margen, $32,0009^{\circ} \mathrm{S} 70,2772^{\circ} \mathrm{W}$, 3628 m s. m., XII-2010, Medina 3344 (CONC!); vega el Circo, $32,0017^{\circ} \mathrm{S} 70,3020^{\circ} \mathrm{W}, 3454 \mathrm{~m} \mathrm{~s}$. m., XII-2010, Medina 3345 (CONC!). Prov. Elqui, valle de Elqui camino hacia Argentina, $30,1648^{\circ} \mathrm{S}$ 70,0309² W, $3441 \mathrm{~m} \mathrm{s.} \mathrm{m.,} \mathrm{19-I-1995,} \mathrm{Ruthsatz} 8930$ (GENT); vega el Tambo, $29,8504^{\circ} \mathrm{S} 69,9404^{\circ} \mathrm{W}$, 3975 m s. m., 08-II-1997, Aron y Edding s. n. (SGO 140491!); estero Derecho vega acojinada curso agua afluente del estero, $30,3810^{\circ} \mathrm{S} 70,3268^{\circ} \mathrm{W}, 3428 \mathrm{~m}$ s. m., 16-III-2017, Medina 3405 (CONC!); ídem, vega acojinada colgante, $30,4108^{\circ} \mathrm{S} 70,2931^{\circ} \mathrm{W}$, 3798 m s. m., 16-III-2017, Medina 3406 (CONC!). Región Metropolitana. Prov. Cordillera, estero la Jarilla afluente río Olivares, $33,3879^{\circ} \mathrm{S} 70,1812^{\circ} \mathrm{W}$, 3151 m s. m., 07-IV-2016, Medina 3334, 3335 (AGUCH 71232!, CONC!); Monumento Natural El Morado laguna de Morales, $33,8000^{\circ} \mathrm{S} 70,0667^{\circ} \mathrm{W}$, 3480 m s. m., 07-III-2002, Teillier \& Márquez 5140 (GENT); río Yeso vegas W. de los baños del Plomo, $33,6181^{\circ} \mathrm{S} 69,9192^{\circ} \mathrm{W}, 2900 \mathrm{~m}$ s. m., 28-I-2009, Teillier et al. 5642. Prov. Santiago, estero Yerba loca en Santuario de la naturaleza Yerba Loca, $33,2205^{\circ} \mathrm{S}$ 70,2744 W, 3243 m s. m., XII-2011, Medina 2566 (SGO!); vega acojinada Piedra Carvajal, Santuario de la naturaleza Yerba Loca, $33,2205^{\circ} \mathrm{S} 70,2744^{\circ} \mathrm{W}$, 3243 m s. m., 10-II-2014, Medina 2611 (SGO 165468 !); ídem, $33,2205^{\circ} \mathrm{S} 70,2744^{\circ} \mathrm{W}, 3243 \mathrm{~m} \mathrm{~s}$. m., VI-2011, Medina 3316, 3317 (AGUCH 71241!, CONC!); ídem, $33,2209^{\circ} \mathrm{S} 70,2739^{\circ} \mathrm{W}, 3259 \mathrm{~m} \mathrm{~s}$. m., 13-XII-2012, Medina 3351 (CONC!); estero Ortiga, $33,1512^{\circ} \mathrm{S} 70,3671^{\circ} \mathrm{W}, 2942 \mathrm{~m}$ s. m., XII-2011, Medina 2723 (SGO 165326!); ídem, $33,1133^{\circ} \mathrm{S} 70,3718^{\circ} \mathrm{W}, 3380 \mathrm{~m}$ s. m., XII-2011, Medina 2729 (SGO 165320!); estero Llaretas, $33,3115^{\circ} \mathrm{S} 70,2220^{\circ} \mathrm{W}, 3055 \mathrm{~m}$ s. m., VI-2011, Medina 3318, 3319 (AGUCH 71240!, CONC!); Los Bronces, $33,1468^{\circ} \mathrm{S} 70,3328^{\circ} \mathrm{W}, 3089 \mathrm{~m} \mathrm{~s}$. m., 03-III-2005, Faúndez s. n. (AGUCH!); ídem, 33, $1468^{\circ} \mathrm{S} 70,3328^{\circ} \mathrm{W}, 3089 \mathrm{~m}$ s. m., 25-III-2005, Esc. s. n. (AGUCH!); ídem, $33,1468^{\circ} \mathrm{S} 70,3328^{\circ} \mathrm{W}$, 3089 m s. m., 02-III-2005, Faúndez s. n. (AGUCH!); ídem, 33,1468 ${ }^{\circ} \mathrm{S} 70,3328^{\circ} \mathrm{W}, 3089$ m s. m., 03III-2005, Faúndez s. n. (AGUCH!); 33,1468 $\mathrm{S}$ 70,3328 ${ }^{\circ} \mathrm{W}, 3089$ m s. m., 04-III-2005, Faúndez s. n. (AGUCH!); ídem, $33,1468^{\circ} \mathrm{S} 70,3328^{\circ} \mathrm{W}, 3089$ m s. m., 26-III-2005, Faúndez s. n. (AGUCH!). Región de Valparaíso. Prov. Los Andes, estero Riecillos, 33,0519 ${ }^{\circ} \mathrm{S} 70,3763^{\circ} \mathrm{W}, 3003 \mathrm{~m} \mathrm{~s} . \mathrm{m}$., XII-2011, Medina 2587 (SGO!); ídem, 33,0519 $\mathrm{S}$ 70,3763 W, 3213 m s. m., XII-2011, Medina 2744 (SGO 165310!). Región del Maule. Prov. Talca, laguna del Maule, $36,0009^{\circ} \mathrm{S} 70,4331^{\circ} \mathrm{W}, 2200 \mathrm{~m}$ s. m., 27-XII-2007, Faúndez et al. s. n. (AGUCH!); ídem, 36,0001 ${ }^{\circ} \mathrm{S} 70,4036^{\circ} \mathrm{W}, 2464 \mathrm{~m} \mathrm{~s}$. m., $27-$ XII-2008, Faúndez et al. s. n. (AGUCH!); ídem, $35,9909^{\circ} \mathrm{S} 70,4006^{\circ} \mathrm{W}, 2496 \mathrm{~m} \mathrm{~s}$. m., 27-XII-2009, Faúndez et al. s. n. (AGUCH!); ídem, 35,9839 ${ }^{\circ} \mathrm{S}$ 70,3952 ${ }^{\circ} \mathrm{W}, 2552 \mathrm{~m} \mathrm{s.} \mathrm{m.,} \mathrm{27-XII-2010,} \mathrm{Faúndez} \mathrm{et}$ al. s. n. (AGUCH!).

Zameioscirpus muticus Dhooge \& Goetgh., Pl. Syst. Evol. 246: 133. 2004. TIPO: Perú, Ancash, sur de Huaraz, $32 \mathrm{~km}$ desde Pachacoto hacia La Union, Punta Huarapasca, 4750 m s.m., 6-IV1988, S. Renvoize \& S. Laegaard 5144 (holotipo GENT cb 150764!).

Nombres vernáculos. No conocidos en Chile; a este tipo de hierbas propias de humedales altoandinos se les denomina genéricamente " $t$ 'champa, t'champita" o vega (Villagrán \& Castro, 2004).

Referencias. Dhooge et al. (2003, 2004), Dhooge (2005).

Distribución geográfica y hábitat. Crece en los humedales altoandinos de Argentina, Bolivia, Chile y Perú, en elevaciones que van entre 4000 - $4800 \mathrm{~m}$ s. m. (Guaglianone, 1996; Dhooge, 2005; Zuloaga et al., 2008; Rodríguez et al., 2018). En la Argentina se extiende entre las provincias de Jujuy y San Juan (Guaglianone, 1996; Dhooge et al., 2003, 2004; Dhooge, 2005; Zuloaga et al., 2008); para Bolivia se citan registros en humedales montañosos de La Paz, Oruro y Potosí (Dhooge et al., 2003, 2004; Dhooge, 2005; Jørgensen et al., 2014); en Perú se ha registrado en los departamentos de Ancash y Lima (Dhooge et al., 2003, 2004; Dhooge, 2005). La distribución en Chile se restringe a humedales altoandinos del extremo norte de Chile, desde la Región de Arica 
y Parinacota hasta la de Antofagasta, entre 4100 4500 m s. m. (Dhooge et al., 2003, 2004; Dhooge, 2005; Rodríguez et al., 2018). En esta contribución se complementa la corología de la especie, mediante la adición de nuevas localidades para el rango de distribución señalado (Fig. 3).

Categoría de conservación. Especie sin protección en la normativa de Chile. No ha sido sometida al proceso de evaluación definido por el Reglamento de Clasificación de Especies del Ministerio de Medio Ambiente de Chile. Ha sido incluida en el catálogo de flora amenazada para la Región de Tarapacá bajo la categoría "Datos Insuficientes" (Gatica-Castro et al., 2015).

\section{Material representativo examinado}

CHILE. Región de Arica y Parinacota. Prov. Parinacota, humedal de Caquena, $18,0214^{\circ} \mathrm{S}$ 69,2149 ${ }^{\circ}$ W, 4371 m s. m., 22-XI-2009, Saldivia 418 (AGUCH!); humedal de Caquena Este, $18,0604^{\circ} \mathrm{S}$ $69,1846^{\circ} \mathrm{W}, 4460 \mathrm{~m}$ s. m., 22-XI-2009, Saldivia 404 (AGUCH!); salar de Surire, bofedal colgante cerca de refugio CONAF, $18,8545^{\circ} \mathrm{S} 69,1427^{\circ} \mathrm{W}, 4290$ m s. m., IV-2010, Medina 3346 (CONC!); ídem, $18,8545^{\circ} \mathrm{S} 69,1427^{\circ} \mathrm{W}, 4290 \mathrm{~m}$ s. m., IV-2011, Medina 3322, 3323 (AGUCH 71238!, CONC!); bofedal de Parinacota, $18,1949^{\circ} \mathrm{S} 69,2971^{\circ} \mathrm{W}, 4408$ m s. m., 10-III-1980, Troncoso s. $n$. (SGO 99953!).
Región de Tarapacá. Prov. Del Tamarugal, Collahuasi, $20,9893^{\circ} \mathrm{S} 68,6507^{\circ} \mathrm{W}, 4435 \mathrm{~m}$ s. m., VIII-2010, Medina 3332, 3333 (AGUCH 71233 !, CONC!); río Aravilla, oberlauf, $19,2274^{\circ} \mathrm{S}$ $68,8552^{\circ} \mathrm{W}, 4120 \mathrm{~m}$ s. m., 28-III-1992, Ruthsatz 8501 (GENT); Lirima, $19,7800^{\circ} \mathrm{S} 68,8177^{\circ} \mathrm{W}, 4551$ m s. m., 12-I-2012, Saldivia 1365 (AGUCH!); ídem, $19,7800^{\circ} \mathrm{S} 68,8186^{\circ} \mathrm{W}, 4544 \mathrm{~m}$ s. m., 12-I-2012, Saldivia 1355 (AGUCH!). Región de Antofagasta. Prov. El Loa, El Tatio Sur, 22,3796 ${ }^{\circ} \mathrm{S} 68,0325^{\circ} \mathrm{W}$, 4314 m s. m., 30-I-1993, Faúndez s. n. (AGUCH!); humedal Pampa Puno, $21,2949^{\circ} \mathrm{S} 68,7725^{\circ} \mathrm{W}, 4050$ m s. m., 13-III-2004, Faúndez s. n. (AGUCH!); vegas del río Zapaleri, $22,9531^{\circ} \mathrm{S} 67,2272^{\circ} \mathrm{W}, 4170$ m s. m., 18-XII-1996, Moreira y Мuñoz 261 (SGO 140645 !); aguada Linzor, $22,2287^{\circ} \mathrm{S} 68,0317^{\circ} \mathrm{W}$; 4300 m s. m., 1981, Villagrán 2989 (CONC 54978!); bofedal al sur de Putana, $22,5305^{\circ} \mathrm{S} 68,0234^{\circ} \mathrm{W}$, 4252 m s. m., 19-III-1992, Ruthsatz 8344 (GENT). Región de Atacama. Prov. Copiapó, quebrada Caspiche, $27,6822^{\circ} \mathrm{S} 69,3110^{\circ} \mathrm{W}, 4157 \mathrm{~m} \mathrm{~s} . \mathrm{m} ., 14-$ II-2012, Canto y Carrasco AC-MC 25 (AGUCH!); ídem, $27,6826^{\circ} \mathrm{S} 69,3235^{\circ} \mathrm{W}, 4093 \mathrm{~m} \mathrm{~s}$. m., 15 -II2012, Canto y Carrasco AC-MC 70 (AGUCH!); punto medio quebrada La Lajita, $27,2817^{\circ} \mathrm{S}$ 68,9656 ${ }^{\circ} \mathrm{W}, 4238 \mathrm{~m}$ s. m., 19-XII-2009, Faúndez et al. s. n. (AGUCH!); Caspiche, cruce camino a Maricunga, $27,6728^{\circ} \mathrm{S} 69,3270^{\circ} \mathrm{W}, 3933 \mathrm{~m} \mathrm{~s}$. m., XII-2009, Faúndez s. n. (AGUCH!).

\section{Clave artificial para el reconocimiento de los géneros de Cyperaceae de Chile continental e insular}

1. Base del aquenio 3-alada y base del estilo 3-alada o abruptamente 3-angulada. Plantas representadas en Isla Robinson Crusoe y zonas tropicales del noreste de Sudamérica (norte de Sudamérica, Guyana, sudeste de Brasil) ............. Machaerina 1. Base del aquenio no 3-alada, base del estilo no 3-alada ni abruptamente 3-angulada. Plantas representadas en Chile continental-insular y Sudamérica

2(1). Flores unisexuales, periginio envuelve al aquenio en forma de utrículo: raquilla secundaria sale por la boca del utrículo y termina en forma de gancho, espiga única terminal, ebracteada (Carex pro parte, incl. Uncinia s.str.) o raquilla secundaria no sale por la boca del utrículo (excepto Carex microglochin y C. camptoglochin), inflorescencia variada, monocéfala, espiga o panícula (Carex pro parte, excl. Uncinia s.str.) Carex

2. Flores perfectas, raro unisexuales o funcionalmente unisexuales por aborto de estructuras, aquenios no envueltos o desnudos 3

3(2). Base del estilo (estilopodio) presente, engrosada y persistente ............................................................... 4

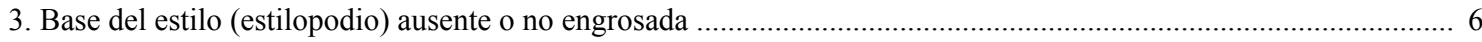

4(3). Plantas áfilas. Inflorescencia única terminal ................................................................................ Eleocharis 4. Plantas con la lámina foliar desarrollada. Inflorescencia pseudolateral con más de una espiguilla a numerosas, raras veces reducida a una espiguilla solitaria 5 5(4). Vaina foliar membranácea, largamente ciliada o fimbriada en su abertura apical, con pilosidad laxa Bulbostylis 
5. Vaina foliar sin pelos en su abertura apical Rhynchospora

6(3). Perigonio presente en forma de setas o cerdas

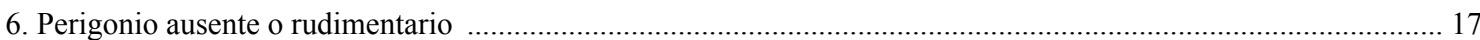

7(6). Perigonio en forma de setas sepaloides o plumosas ................................................................................. 8

7. Perigonio en forma de cerdas lisas o escábridas de tamaño variable alcanzando más de $1 / 3$ del aquenio o superándolo ..... 9

8(7). Pseudoespiga con flor única. Perigonio en forma de setas sepaloides, más breves que el aquenio .............. Oreobolus

8. Pseudoespiga con más de una flor fértil (1-3). Perigonio en forma de setas plumosas, más largas que el aquenio ..... Carpha

9(7). Plantas acaules. Rosetas adpresas que provienen de rizomas verticales ......................................... Phylloscirpus

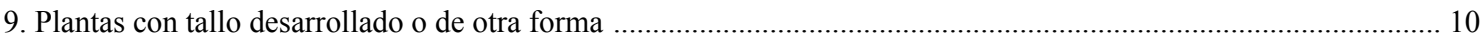

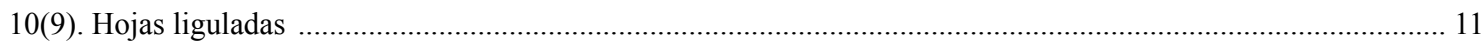

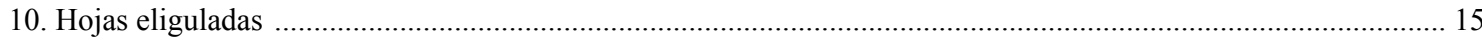

11(10). Tallos teretes. Hojas rígidas. Glumas ovadas con margen ciliado. Perigonio en forma de cerdas retrorso híspidas, más cortas que el aquenio

Amphiscirpus

11. Tallos de sección trígona. Hojas gráciles. Glumas de margen entero. Perigonio en forma de cerdas escábridas del tamaño del aquenio o más largas

12

12(11). Hojas escasas, involutas o sección en forma de "v", rígidas. Inflorescencias sésiles, contraídas o capitadas.

Glumas de ápice bilobado a emarginado, de borde entero con carena marcada, terminando en mucrón apical prolongado.

Aquenios de 1,8-2,1 mm

12. Tallos con numerosas hojas, aplanadas, de consistencia papirácea. Inflorescencias contraídas a ramificadas, glumas de ápice entero, carinadas, sin mucrón prolongado. Aquenios menores a $1,8 \mathrm{~mm}$............................................... 14 13(12). Nervadura central de la gluma prolongada en un mucrón breve de 0,1 a $0,7 \mathrm{~mm}$, más corto o ligeramente más largo que los lóbulos del ápice Schoenoplectus americanus 13. Nervadura central de la gluma prolongada en un mucrón de 1 a $2 \mathrm{~mm}$, más larga que los lóbulos del ápice, algo curvada Schoenoplectus pungens

14(12). Inflorescencia contraída, terminal, escasamente ramificada en antelodio. Estigma generalmente bífido ........ Scirpus 14. Inflorescencia en antelodio compuesto por 4-12 paracladios, rara vez contraída. Estigma trífido ...... Rhodoscirpus 15(10). Tallos teretes. Inflorescencia capitada. Glumas dísticas en la espiguilla, lanceoladas, de ápice agudo, carinadonaviculares aquilladas, de margen entero. Estigma trífido Schoenus 15. Tallos de sección trígona, subtrígona a terete. Inflorescencia subsésil a ramificada. Glumas espiraladas en la espiguilla, margen escabroso-ciliado a antrorso escábrido. Estigma 2(3)-fido 16 16(15). Tallos trígonos a teretes. Hojas reducidas a la vaina. Inflorescencia contraída a ramificada, nutante. Glumas ampliamente ovadas a ovadas, ápice obtuso a agudo, margen escabroso-ciliado, cerdas del perigonio reducidas a 2-4 escamas, más cortas que el cuerpo del aquenio, aplanadas, rojo oscuro, lineares, irregularmente plumosas. Estigma bífido Schoenoplectus californicus

16. Tallos trígonos, hojas generalmente numerosas. Inflorescencias subsésil a brevemente pedunculada. Glumas de ápice asimétricamente emarginado y mucronado, divisiones de los dientes agudo-aristadas, margen antrorso-escábrido, cerdas del perigonio desarrolladas, del tamaño del aquenio o ligeramente menor, caducas. Estigma 2(3)-fido Bolboschoenus 17(6). Perigonio rudimentario o reducido, formando un breve ginóforo. Glumas sin carena marcada. Estigma trífido .... Ficinia 17. Perigonio ausente. Glumas con carena marcada. Estigma bífido o trífido ……….................................................... 18

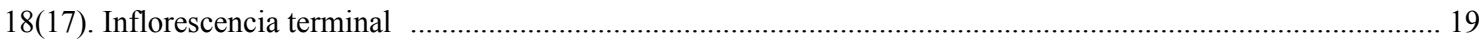

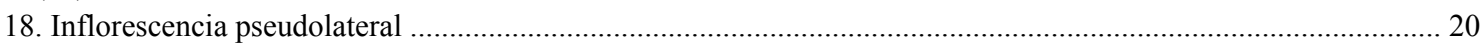

19(18). Hierbas perennes, rizomatozas, con hojas. Inflorescencia uniespiculada, pedunculada, terminal. Plantas que habitan en humedales altoandinos por encima de los $3000 \mathrm{~m} \mathrm{s.m.}$

Zameioscirpus

19. Hierbas anuales, generalmente áfilas o con hojas reducidas. Inflorescencias con 1 a 3 espiguillas sésiles. Plantas que habitan en humedales por debajo de los $3000 \mathrm{~m} \mathrm{s.m.}$ Isolepis

20(18). Glumas dispuestas en forma dística en la raquilla. Inflorescencia generalmente con más de 3 espiguillas Cyperus (incl. Pycreus)

20. Glumas dispuestas de manera espiralada o de otra forma en la raquilla. Inflorescencia con 1 a 3 espiguillas 


\section{DISCUSIÓN}

Este estudio documenta dos nuevos registros para la flora ciperológica de Chile y proporciona nueva información geográfica de diversas Cyperaceae altoandinas, lo que permite extender y complementar sus rangos de distribución. Estos hallazgos evidencian el conocimiento actual insuficiente a nivel nacional para la familia Cyperaceae, sobre todo para especies que componen comunidades de humedales altoandinos. Esta situación se debe en parte al reducido número de botánicos que recolectan material y estudian esta familia, lo que se traduce para la mayoría de los taxa en areales fragmentados, incompletos y desactualizados (Marticorena, 1990; Marticorena et al., 1998b; Squeo et al., 2001, 2008; Dhooge et al., 2003; Dhooge, 2005; Zuloaga et al. 2008; García, 2013; Gatica-Castro et al., 2015; Rodríguez et al., 2018; Govaerts et al., 2019). Esto redunda en la reducida cantidad de material depositado en herbarios de Chile (CONC, EIF, SGO y ULS), en comparación con la considerable representación de especímenes recolectado en Chile y que se encuentran en herbarios fuera del país, como por ejemplo, BA, BAB, GENT, GH, K, SI, NY, US y WU (Dhooge, 2005). De acuerdo a lo antes mencionado, conocimiento actual en de Cyperaceae en Chile es insuficiente y se necesitan más trabajos para extender y completar el conocimiento de esta familia.

En relación a Zameioscirpus gaimardioides y Z. muticus, a partir de evidencia de campo, observación de ejemplares de herbario y literatura (Dhooge et al., 2003; Dhooge, 2005), se puede indicar que ambas especies son taxa afines y en términos generales alopátricos, aunque con una zona de sobreposición (simpatría) en la zona altoandina de la región de Atacama (Fig. 3). Sin embargo, las diferencias morfológicas entre individuos de ambas especies distribuidos en esta zona de sobreposición son ambiguas, poco notorias y se traslapan. Los principales caracteres que señalan Dhooge et al. (2003) para separar las dos especies son tamaño de las espiguillas, la prolongación del internudo de la raquilla (acortado e inconspicuo vs. elongado y visible, respectivamente) y el número de glumas (4-6 vs. 3-5, respectivamente) y la forma del fruto (angostamente obovada a obovada vs. obovado, respectivamente). Tomando en consideración el reducido número de ejemplares citados por Dhooge et al. (2003) (3 especímenes de Z. gaimardioides y 5 de $Z$. muticus) y que ninguno de ellos proviene de la zona en cuestión, se sugiere llevar a cabo mayores estudios morfométricos y moleculares para evaluar la asignación taxonómica de $Z$. muticus. La evidencia molecular de Dhooge et al. (2003) basada en dos marcadores cloroplásticos señalan que ambas son especies hermanas, lo que se podría también interpretar como una sola especie variable morfológicamente, o bien dos especies muy relacionadas con una zona de hibridación debido a sobreposición dentro del patrón de distribución.

\section{AGRADECIMIENTOS}

A las curadoras de los Herbarios CONC, SGO, ULS, Alicia Marticorena, Gloria Rojas y Gina Arancio, respectivamente, por la proporción de información y comentarios. A Barbara Ruthsatz, por el envío de datos y georeferencia de especímenes depositados en SI, GENT, NY, US. A Kenneth Bauters (GENT) y Mats Hjertson (UPS) por envío de material tipo digitalizado de Zameioscirpus muticus y Cyperus fuscus, respectivamente. A Gonzalo Moreno por la información brindada. A los revisores anónimos por sus comentarios y mejoras al documento.

\section{BIBLIOGRAFÍA}

Adams, C. D. 1994. Pycreus, en G. Davidse, M. Sousa-Sánchez \& A. O. Chater (eds.), Flora Mesoamericana; Alismataceae a Cyperaceae. Vol 6, pp. 440-442. Universidad Nacional Autónoma de México, México, D. F. 543 pp.

Alfaro, V. \& L. Faúndez. 2010. Caracterización base de vegas y bofedales altoandinos para una gestión sostenible de los recursos hídricos. Primera parte: segunda Región de Antofagasta. Proyecto Innova Chile. 41 pp.

Ball, P. W.; A. A. Reznicek \& D. F. Murray. 2002. Cyperaceae, en Flora of North America Editorial Committee (eds.), Flora of North America, Magnoliophyta: Commelinidae (in part) Vol. 23: 1-608. Oxford University Press. New York, United States.

Barros, M. 1938. Ciperáceas Argentinas III. Géneros Androtrichum, Lipocarpha, Ascolepis, Cyperus. Anales del Museo Argentino Ciencias Naturales Bernardino Rivadavia 85. 39: 253-381.

Barros, M. 1941. Distribución geográfica de las Ciperáceas Argentinas. Darwiniana 5: 41-46. 


\section{P. MEDINA, P. SALDIVIA \& L. FAÚNDEZ. Nuevas citas y aportes a Cyperaceae de Chile}

Barros, M. 1945. Ciperáceas argentinas IV. Géneros Fymbristylis, Bulbostylis, Fuirena, Dichromena, Schoenus, Oreobolus, Carpha, Rhynchospora, Scleria y Uncinia. Anales del Museo Argentino Ciencias Naturales Bernardino Rivadavia 87. 41: 323-479.

Barros, M. 1969. Cyperaceae, en M. N. Correa (ed.), Flora Patagónica, Colección Cientifica del Instituto Nacional de Tecnología Agropecuaria 8(2): 38-92.

Bernardello, G.; G. J. Anderson, T. F. Stuessy \& D. Crawford. 2006. The angiosperm flora of the Juan Fernández Archipiélago (Chile): origin and dispersal. Canadian Journal of Botany 48: 1266-1281.

Boeckeler, O. 1867. Die Cyperaceen des Königlichen herbariums zu Berlin. Journal für die Botanik in ihrem ganzen Umfange 35: 397-612.

Castroviejo, S. 2008. Cyperus, en S. Castroviejo, S., C. Aedo, M. Laénz, F. Muñoz-Garmendia, G. Nieto-Feliner, J. Paiva \& C. Benedí (eds.), Flora Ibérica. Vol 18: 8-27. Real Jardín Botánico, CSIC, Madrid.

Corcoran, M. L. 1941. A revision of the subgenus Pycreus in North and South America. The Catholic University of America, Biological Series 37: 1-68.

Costé, A. 1937. Flore descriptive et ilustreé de la France de la corse et des contrées limitrophes. Tomé III. Second tirage. Paris, France. 728 pp.

Dhooge, S. \& P. Goetghebeur. 2004a. Phylloscirpus (Cyperaceae) Revisited. Novon 14(3): 278-284.

Dhooge, S. \& P. Goetghebeur. 2004b. Two new subspecies in Andean Phylloscirpus and Trichophorum (Cyperaceae). Nordic Journal of Botany 24(4): 399-402.

Dhooge, S.; P. Goetghebeur \& A. M. Muasya. 2003. Zameioscirpus, a New Genus of Cyperaceae from South America. Plant Systematics and Evolution 243: 73-84.

Dhooge, S.; P. Goetghebeur \& A. M. Muasya. 2004. Erratum. Plant Systematics and Evolution 246: 133.

Dhooge, S. 2005. Systematic Revision and Phylogeny of the Andean Scirpoids (Cyperaceae, Scirpeae). Tesis Doctoral. University of Gent, Belgium. 240 p.

Dai, L.; G. C. Tucker \& D. Simpson. 2010. Cyperus, en C. Y. Wu, P. H. Raven \& D. Y. Hong (eds.), Flora of China (Acoraceae through Cyperaceae). Vol 23: 219-241. Press \& Missouri Botanical Garden Press, Beijing \& St. Louis.

García P. 2013. Estado de conservación de la flora vascular de la Región de Antofagasta, Chile. Tesis de MSc. en Ciencias Biológicas mención Ecología de Zonas Áridas, Universidad de La Serena, Chile. 126 pp.

Gatica-Castro, A.; A. Marticorena, G. Rojas, G. Arancio \& F. A. Squeo. 2015. Estado de conservación de la flora nativa de las regiones de Arica-Parinacota y de Tarapacá, Chile. Gayana Botánica 72(2): 305-339.
Global Carex Group. 2015. Making Carex monophyletic (Cyperaceae, tribe Cariceae): a new broader circumscription. Botanical Journal of the Linnean Society 179: 1-42.

Goetghebeur, P. \& D. Simpson. 1991. Critical Notes on Actinoscirpus, Bolboschoenus, Isolepis, Phylloscirpus and Amphiscirpus (Cyperaceae). Kew Bulletin 46(1): 169-178.

Goetghebeur, P. 1998. Cyperaceae, in K. Kubitzki (ed.), The Families and Genera of Vascular Plants 4. Flowering Plants Monocotyledons: Alismatanae and Commelinanae (except Gramineae), pp. 141-190. Springer, Berlin Heidelberg New York.

Govaerts, R.; D. A. Simpson, P. Goetghebeur, K. L. Wilson, T. Egorova \& J. Bruhl. 2019. World checklist of Selected Plant Families: Cyperaceae. Facilitated by the Royal Botanic Gardens, Kew. Published on the Internet: http://apps.kew. org/wcsp/ [consulta febrero 2019].

Guaglianone E. R. 1996. Cyperaceae, en: Zuloaga F. O. \& M. Morrone (eds.). Catálogo de las plantas vasculares de la República Argentina. I. Monographs in Systematic Botany from the Missouri Botanical Garden 60: 128-197.

Guaglianone E. R.; O. Ueno \& B. Ruthsatz. 1998. Note on Eleocharis tucumanensis (Cyperaceae). Darwiniana 35: 169-173.

Guaglianone, E. R. \& M. S. González-Elizondo. 2009. Eleocharis, en R. Kiesling (Dir.), Flora de San Juan 4: 299-312. Editorial Fundación Universidad Nacional de San Juan, República Argentina.

Guaglianone, E. R.; G. Wheeler \& M. S. González-Elizondo. 2009. Cyperaceae, en R. Kiesling (Dir.), Flora de San Juan 4: 287-338. Editorial Fundación Universidad Nacional de San Juan, República Argentina.

Jørgensen P. M.; M. H. Nee \& S. G. Beck (eds.). 2014. Catálogo de las plantas vasculares de Bolivia. Monographs in Systematic Botany from the Missouri Botanical Garden 127 (1-2): i-viii, 1-1744. Missouri Botanical Garden Press, St. Louis.

Koyama, T. 1974. New combinations relevant to the Cyperaceae tribe Cypereae of tropical America. Phytologia 29: 73-74.

Kükenthal, G. 1935-36. Cyperaceae - Scirpoideae - Cypereae, en A. Engler (ed.), Das Pflanzenreich 4 (20) [Heft 101], pp. 1-671. Engelmann, Berlin.

Lansdown, R. V. 2013. Cyperus fuscus. The IUCN Red List of Threatened Species 2013: e.T164079A13545918. DOI: 10.2305/IUCN.UK.2013-1.RLTS.T164079A13545918.en

Larridon, I.; M. Reynders, W. Huygh, K. Bauters, K. Van de Putte, A. M. Muasya, P. Boeckx, D. A. Simpson, A. Vrijdaghs \& P. Goetghebeur. 2011. Affinities in C3 Cyperus lineages (Cyperaceae) revealed using molecular phylogenetic data and carbon isotope analysis. Botanical Journal of the Linnean Society 167: 19-46. 
Luceño, M.; S. Castroviejo \& P. Jiménez-Mejías. 2008. Cyperaceae, en S. Castroviejo, C. Aedo, M. Laénz, F. Muñoz-Garmendia, G. Nieto-Feliner, J. Paiva \& C. Benedí (eds.). Flora Ibérica 18: 3-250. Real Jardín Botánico, CSIC, Madrid. 472 pp.

Luebert F. \& R. Gajardo. 2004. Antecedentes sobre la vegetación de la cordillera de los Patos, Andes de Chile central (Región de Valparaíso, V). Chloris Chilensis Año 7. N ${ }^{\circ}$ 2. URL: http://www.chlorischile.cl [consulta junio 2018].

Luebert F. \& P. Pliscoff. 2006. Sinopsis bioclimática y vegetacional de Chile. Editorial Universitaria, Santiago. 316 pp.

Marticorena C. 1990. Contribución a la estadística de la flora vascular de Chile. Gayana Botánica 47(3-4): 85-113.

Marticorena, C.; T. F. Stuessy \& C. Baeza. 1998a. Catalogue of the vascular flora of the Robinson Crusoe or Juan Fernández islands, Chile. Gayana Botánica 55: 187-211.

Marticorena C.; O. Matthei, R. Rodríguez, M. T. K. Arroyo, M. Muñoz, F. A. Squeo \& G. Arancio. 1998b. Catálogo de la flora vascular de la Segunda Región (Región de Antofagasta), Chile. Gayana Botánica 55: 23-83.

MacBride, J. F. 1936. Cyperus, en J. F. MacBride \& B. H. Dahlgren (eds.), Flora of Peru. Field Museum of Natural history-Botany. Publication 351. 13(1/1): 264-278. Chicago, United States.

McGivney, M. 1938. A revision of the subgenus Eucyperus found in the United States. The Catholic university of America, biological series 26: 1-76.

McKenzie, P.; B. Jacobs, C. Bryson, G. Tucker \& R. Carter. 1998. Cyperus fuscus (Cyperaceae), new to Missouri and Nevada, with comments on its ocurrence in North America. SIDA 18(1): 325-333

Medina, P. 2015. Eleocharis tucumanensis Barros (Cyperaceae) en Chile central, ampliación de distribución y contribución al conocimiento de la especie. Lilloa 52(2): 175-186.

Moreira-Muñoz A. 2012. Plant geography of Chile. Primera Edición. Springer, Germany. 320 pp.

Oteng-Yeboah, A. 1974. Four new genera on CyperaceaeCyperoideae. Notes from the Royal Botanical Garden 33: 307-310.

QGIS Development Team. (2019). QGIS Geographic Information System. Open Source Geospatial Foundation. Published on the Internet: https://www.qgis.org/es/site/ [consulta marzo 2019].
Rodríguez, R.; C. Marticorena, D. Alarcón, C. Baeza, L. Cavieres, V. L. Finot, N. Fuentes, A. Kiessling, M. Mihoc, A. Pauchard, E. Ruiz, P. Sánchez \& A. Marticorena. 2018. Catálogo de las plantas vasculares de Chile. Gayana. Botanica 75 (1): 1-430.

Serey, I.; M. Ricci \& C. Smith-Ramírez (eds.). 2007. Libro Rojo de la Región de O'Higgins. CONAF - Universidad de Chile. $206 \mathrm{pp}$.

Smith, S. G. 2002. Amphiscirpus, en Flora of North America Editorial Committee (eds.), Flora of North America North of Mexico, Magnoliophyta: Commelinidae (in part) Vol 23: 27-28. Oxford University Press, New York.

Squeo, F.; G. Arancio \& J. Gutiérrez (eds.). 2001. Libro Rojo de la Flora Nativa y de los Sitios Prioritarios para su Conservación: Región de Coquimbo. Ediciones Universidad de La Serena, La Serena. 372 pp.

Squeo, F.; G. Arancio \& J. Gutiérrez (eds.). 2008. Libro Rojo de la Flora Nativa y de los Sitios Prioritarios para su Conservación; Región de Atacama. Ediciones Universidad de la Serena. Primera Edición. La Serena, Chile. 466 p.

Strong, M. 1997. Machaerina (Cyperaceae) in South America. Novon 7(3): 308-319.

Thiers, B. [permanentemente actualizado, consulta marzo 2019] Index Herbariorum: a global directory of public herbaria and associated staff. New York Botanical Garden's Virtual Herbarium, http://sweetgum.nybg.org/ih

Tucker, G. 1983. The taxonomy of Cyperus (Cyperaceae) in Costa Rica and Panama. Systematic Botany Monographs 2: 1-85.

Tucker, G. 1994. Revision of the Mexican species of Cyperus (Cyperaceae). Systematic Botany Monographs 43: 1-213.

Tucker, G.; B. Marcks \& R. Carter. 2002. Cyperus L., en Flora of North America Editorial Committee (eds.), Flora of North America, Magnoliophyta: Commelinidae (in part) Vol 23: 141-191. Oxford University Press. New York, United States.

Villagrán C. \& V. Castro. 2004. Ciencia indigena de los Andes del norte de Chile. Editorial Universitaria, Santiago, Chile. 362 pp.

Zuloaga, F. O.; O. Morrone \& M. Belgrano (eds.). 2008. Catálogo de las Plantas Vasculares del Cono Sur (Argentina, Sur de Brasil, Chile, Paraguay y Uruguay), vol. 1. Pteridophyta, Gymnospermae y Monocotyledoneae. Monograph of Systematic Botany from Missouri Botanical Garden 107(1): 1-161. 